ARTIGO ORIGINAL

ORIGINAL ARTICLE

\section{Radioterapia intraoperatória com elétrons no tratamento do câncer colorretal localmente avançado submetido a ressecção incompleta: análise econômica sob a perspectiva do Sistema de Saúde Suplementar brasileiro}

\author{
Intraoperative electron-beam radiotherapy in locally \\ advanced rectal cancer, in patients submitted to \\ suboptimal surgery: economic analysis on the \\ Brazilian Private Health Care system perspective
}

Marcos Santos ${ }^{1}$

DOI: 10.21115/JBES.v12.n1.p66-70

\section{RESUMO}

radioterapia intraoperatória, câncer de reto, controle local

\section{Keywords:}

intraoperative radiotherapy, rectum cancer, local control
Objetivo: Este estudo objetivou avaliar o custo-efetividade da aplicação da radioterapia intraoperatória com elétrons (RIOe) no paciente com câncer retal localmente avançado (CRLA) submetido a radio/quimioterapia neoadjuvante seguida de ressecção incompleta (margem comprometida ou doença grosseira residual). Métodos: Para tanto, foi elaborado um modelo de Markov. Os dados de eficácia, efeitos secundários, controle local e sobrevida global foram extraídos da literatura. A perspectiva considerada foi a do sistema de saúde privado brasileiro. Considerou-se, para o custo e para a efetividade, uma taxa de desconto anual de 5\%. Resultados: A estratégia que continha a RIOe mostrou-se, nesse cenário, dominante em comparação à estratégia que não envolvia essa abordagem. Os resultados mostraram-se robustos após análises de sensibilidade uni e multivariadas. Conclusão: Concluiu-se que pode ser vantajosa, tanto para os pacientes com CRLA quanto para as operadoras de saúde do mercado privado brasileiro, a aplicação de RIOe na ocorrência de cirurgia subótima, ainda que se sugira que esses dados devem ser confirmados posteriormente, por meio da coleta de dados de mundo real, devido à ausência de níveis robustos de evidência, na literatura, para esse cenário clínico específico.

\begin{abstract}
Objective: This study aimed to evaluate the cost-effectiveness of intraoperative electron radiotherapy (IOeRT) in patients with locally advanced rectal cancer (LARC) undergoing neoadjuvant radio-chemotherapy, followed by incomplete resection (compromised margin or gross residual disease). Methods: A Markov model was constructed. Data on efficacy, side effects, local control and overall survival were extracted from the literature. The perspective was that of the Brazilian private health system. For the cost and effectiveness, an annual discount rate of 5\% was considered. Results: The strategy that contained IOeRT was, in this scenario, dominant, in comparison to standard of care. Results were considered robust, after univariate and multivariate sensitivity analyzes. Conclusions: We concluded that it can be advantageous, both for patients with LARC submitted to suboptimal surgery, as well as for Brazilian health insurance companies, the application of IOeRT. Although it is suggested that these results should be confirmed later, through the collection of real-world data, due to the absence of randomized controlled trials, in this specific clinical scenario.
\end{abstract}

Recebido em: 20/02/2020. Aprovado para publicação em: 06/03/2020

1. Cátedra Unesco de Bioética - Universidade de Brasília, Brasília, DF, Brasil,

Local onde o estudo foi conduzido: Cátedra Unesco de Bioética - Universidade de Brasília,

Campus Universitário Darcy Ribeiro, Faculdade de Ciências da Saúde, Brasília, DF, Brasil.

Fontes de financiamento: $\mathrm{O}$ artigo não recebeu financiamento.

Conflito de interesses: $O$ autor não tem conflito de interesses a declarar.

Autor correspondente: Marcos Santos. Cátedra Unesco de Bioética - Universidade de Brasília, Campus Universitário Darcy Ribeiro, Faculdade de Ciências da Saúde - Caixa Postal 04451. Brasília, DF, Brasil. CEP: 70919-970. E-mail: marcosrxt@gmail.com 


\section{Introdução}

A mais recente estimativa epidemiológica do câncer colorretal aponta para a ocorrência de, aproximadamente, 1 milhão de casos anuais entre os homens e 800 mil, entre as mulheres, em todo o mundo. As maiores incidências são encontradas na Europa (Hungria, Eslovênia, Eslováquia, Holanda e Noruega), Austrália/Nova Zelândia, América do Norte e Leste da Ásia (Bray et al., 2018). Estima-se que, no Brasil, para cada ano do triênio 2020-2022, mais de 41 mil casos dessas neoplasias serão diagnosticados, o que corresponde a um risco estimado de pouco mais de 19 casos para cada 100 mil habitantes. Entre os homens, desconsiderados os tumores de pele, é o segundo mais frequente no Sudeste e Centro-Oeste; já entre as mulheres, o mesmo ocorre nas regiões Sudeste e Sul (Inca, 2019).

Desde a primeira publicação de Sauer et al., em 2004 (Sauer et al., 2004), posteriormente atualizada (Sauer et al., 2012), o tratamento-padrão para o câncer retal localmente avançado (CRLA) compõe-se de radioterapia neoadjuvante (50,4 Gy em 28 frações dirigidos à pelve) associada a quimioterapia radiossensibilizante, seguidas de cirurgia (ressecção mesorretal total), preferencialmente após transcorrido um período superior a seis semanas (Calvo et al., 2014). Essa abordagem apresenta controle local superior à estratégia adjuvante, após 10 anos de seguimento, apesar da ausência de diferenças no controle sistêmico da enfermidade ou na sobrevida global dos pacientes (Sauer et al., 2012).

Evidências bem conhecidas apontam no sentido de que a radicalidade da ressecção é o preditor de sobrevida de maior significância clínica (Kusters et al., 2009; Wells et al., 2007). Ainda assim, observa-se uma elevada incidência de recidivas locais nesses indivíduos (Valentini et al., 2011), a maior parte delas na região pré-sacral (Yu et al., 2008). Dados os resultados negativos apresentados por diferentes estratégias de tratamento sistêmico, como, por exemplo, a adição de oxaliplatina às fluoropirimidinas neoadjuvantes, é possível que se tenha, com essa estratégia, atingindo um platô nos resultados, fazendo do escalonamento de dose de radioterapia uma opção interessante nesse cenário (Sole et al., 2014).

A aplicação de doses superiores a 50 Gy com radioterapia externa, seja com técnica conformacional ou com radioterapia de intensidade modulada (IMRT), é dificultada pela dose limite dos órgãos ou tecidos localizados ao redor do alvo do tratamento (Kronborg et al., 2019; Kundel et al., 2020; Owens et al., 2019). Assim sendo, há efetividade no tratamento da enfermidade microscópica, mas a dose é insuficiente para o tratamento de lesões macroscópicas ou metástases linfonodais (Fletcher, 1973). A radioterapia intraoperatória com elétrons (RIOe) tem a vantagem de poder aplicar doses adicionais de radiação em regiões profundas do leito tumoral, onde há elevado risco de presença de células tumorais remanescentes, enquanto órgãos e tecidos sãos são mobilizados, temporariamente, para fora do campo de radiação (Calvo et al., 2006a).
Há evidências crescentes que sugerem que a inclusão da RIOe no tratamento multimodal do CRLA pode levar à melhora do controle local (Mirnezami et al., 2013). No entanto, quando se analisa separadamente o subgrupo de pacientes para os quais foi possível atingir uma ressecção completa, estudos indicam equivalência no controle local, com ou sem RIOe (Ferenschild et al., 2006; Willett et al., 1991), incluso um estudo randomizado, de fase III, francês, com 142 pacientes. O controle local em cinco anos foi semelhante em ambos os grupos (91,8\% com RIOe e 92,8\% sem RIOe), assim como a sobrevida livre de doença e a sobrevida global dos pacientes (Dubois et al., 2011).

Há, no entanto, aquelas situações em que a busca por uma ressecção com margem negativa pode ser questionável, uma vez que o tumor tenha atingido e invadido estruturas vizinhas, tornando a ressecção, quando e se possível, altamente mórbida para o paciente. A aplicação adicional de terapias locais, como a RIOe, pode ser tentada nesse cenário com o objetivo de otimizar o controle local e melhorar o resultado final da terapia oncológica (Roeder et al., 2012). Aqui, a evidência, embora de inferior qualidade (não há, de que tenhamos notícia, estudos randomizados estudando essa condição clínica), aponta no sentido do melhor controle local com a RIOe (Sole et al., 2014).

O objetivo deste estudo foi, então, avaliar a eficácia da RIOe como complemento do tratamento local em pacientes com CRLA submetidos a radioquimioterapia neoadjuvante, seguida de ressecção incompleta (margens comprometidas), e posteriormente avaliar o custo-efetividade dessa estratégia, em comparação ao tratamento neoadjuvante com radio e quimioterapia isolados, sob a perspectiva do sistema de saúde suplementar brasileiro.

\section{Material e métodos}

Foi efetuada uma revisão bibliográfica exaustiva utilizando-se de base de dados PubMed, anais de congressos, registros de estudos, lista de referências de estudos publicados, artigos de revisão e livros relevantes. Não houve restrições no tocante ao idioma dos artigos. No que se refere aos resumos publicados em anais de congressos, os investigadores foram contatados por meio de correio eletrônico em pelo menos duas oportunidades, com o objetivo de recolher informações faltantes em

Tabela 1. Questão de pesquisa estruturada no formato PICO

\begin{tabular}{ll}
\hline População & $\begin{array}{l}\text { Pacientes com neoplasia de reto localmente } \\
\text { avançada submetidos a cirurgia incompleta } \\
\text { após radioquimioterapia neoadjuvante }\end{array}$ \\
\hline Intervenção & $\begin{array}{l}\text { Radioterapia conformada tridimensional } \\
\text { associada a quimioterapia neoadjuvante }+ \\
\text { radioterapia intraoperatória com elétrons }\end{array}$ \\
\hline Comparadores & $\begin{array}{l}\text { Radioterapia conformada tridimensional } \\
\text { associada a quimioterapia neoadjuvante }\end{array}$ \\
\hline Desfechos & Controle local e sobrevida global \\
\hline
\end{tabular}


seus relatos. Ensaios clínicos randomizados publicados eram elegíveis para inclusão. Foram excluídos estudos de fase I ou II. $\mathrm{Na}$ ocorrência de mais de uma publicação, referente ao mesmo grupo de pacientes, a publicação mais recente foi a considerada.

\section{Resultados}

As buscas foram realizadas no mês de março de 2019. Foi avaliada a base de dados PubMed, com os seguintes termos de busca:

("Rectal Neoplasms/radiotherapy"[Mesh] OR
"Rectal Neoplasms/surgery"[Mesh] OR "Rectal
Neoplasms/therapy"[Mesh]) AND "Intraoperative
Radiotherapy"[All Fields] AND "electron"[All Fields]

Não foi acrescentada nenhuma limitação de idioma no processo de busca, que retornou um artigo de Sole et al., publicado no ano de 2014 (Sole et al., 2014).

\section{Descrição do estudo}

Trata-se de uma análise retrospectiva que buscou avaliar os fatores de risco para recorrência locorregional na maior e mais madura coorte de pacientes tratados com RIOe para o diagnóstico de CRLA publicada na literatura. Entre janeiro de 1995 e dezembro de 2010, 335 pacientes com CRLA, em sua maioria do sexo masculino (60\%), com idade variando entre 31 e 86 anos (mediana: 63), foram tratados com 50,4 Gy em 28 frações, com radioterapia externa e quimioterapia radiossensibilizante. Entre os anos de 1995 e 1998, os pacientes foram tratados com 5-FU. Entre 1998 e 2010, 400 mg de tegafur eram administrados por via oral, três vezes ao dia, do D1 ao D28 da radioterapia. De maio de 2001 a 2011, os pacientes receberam, também, dois ciclos de FOLFOX de indução, como parte de um protocolo de pesquisa descrito em outra publicação (Calvo et al., 2006b). A cirurgia era programada para quatro a seis semanas após o término do tratamento combinado. Não houve registro a respeito da excisão total do mesorreto. A RIOe era aplicada em um acelerador linear não dedicado, na dose de 10 a 15 Gy (mediana: 12,5 Gy), em dose única, dirigidos ao espaço pélvico posterior. A dose da RIOe era escolhida baseando-se no tamanho do aplicado, definido de acordo com a anatomia do paciente e, também, na ocorrência (ou não) de ressecção microscopicamente completa (doses superiores, quando da ocorrência de margens comprometidas). Posteriormente, os pacientes receberam quimioterapia adjuvante, quatro a seis ciclos de 5-FU associado a leucovorina (Sole et al., 2014).

Após um seguimento médio de 72,6 meses, 64,5\% estavam vivos. Dos 119 que foram a óbito, 58\% foram por conta de progressão da patologia. Um total de 23 doentes apresentou recidiva local $(6,9 \%)$, sendo 11 dentro e 12 fora do campo de irradiação. Aproximadamente $1 / 4$ dos doentes, ao longo do seguimento, apresentou metástases a distância, sendo o pul- mão e o fígado os órgãos mais atingidos (36 e 33 pacientes, respectivamente). Doze dos pacientes que apresentaram recidiva local foram resgatados com novo procedimento cirúrgico e seis deles estavam vivos ao final do seguimento, que variou, para esses doentes, entre 43 e 109 meses. A sobrevida global de todo o grupo, em 10 anos, foi de $62 \%$. E o controle local, no mesmo período, foi de $92 \%$. As variáveis que estiveram associadas a um melhor controle local foram: ressecção completa, tumor grau I ou II, margem distal igual ou superior a $1 \mathrm{~cm}$ e grau de regressão tumoral elevado.

\section{Análise econômica}

Para a análise econômica, de custo-efetividade, foi construído um modelo de Markov para comparar, em pacientes com diagnóstico de CRLA submetidos a radio/quimioterapia neoadjuvantes e ressecção incompleta, os braços:

- Radio/quimioterapia neoadjuvante seguida de cirurgia e RIOe, com posterior quimioterapia adjuvante;

- Radio/quimioterapia neoadjuvante seguida de cirurgia com posterior quimioterapia adjuvante.

Como não identificamos estudos ou séries de casos de pacientes com CRLA submetidos a radio/quimioterapia neoadjuvante e cirurgia de ressecção incompleta, sem nenhum tratamento local adicional, os dados desse braço do modelo foram extraídos da metanálise de Mirnezami et al., que revisou a literatura considerando como critério de inclusão qualquer radioterapia intraoperatória (e não somente aquela aplicada com elétrons) (Mirnezami et al., 2013). Devido a esse fato, esse primeiro braço (experimental) não foi considerado.

O modelo simulou os possíveis desfechos desses doentes, que podem permanecer livres da recidiva das suas patologias, apresentar recidiva local ou a distância ou, finalmente, progredir para o desfecho letal. Adotaram-se ciclos anuais. Os custos incluíram os procedimentos efetuados para o tratamento inicial e aquele necessário por conta de eventuais recidivas. Valores de reembolso de consultas, exames e procedimentos foram valorados de acordo coma Classificação Brasileira Hierarquizada de Procedimentos Médicos, 5a edição (CBHPM, 2017). Custos de medicamentos foram extraídos da lista da Câmara de Regulação do Mercado de Medicamentos, considerando o preço-fábrica ou o preço máximo de venda ao governo, com 18\% de ICMS, em reais (R\$). Os custos consideraram a média de preços incluindo medicamentos de referência e genéricos disponíveis no mercado (Agência Nacional de Vigilância Sanitária). As probabilidades de transição foram extraídas dos estudos supramencionados. Considerou-se uma taxa de desconto de 5\% ao ano para custos e desfechos. A perspectiva adotada foi a das operadoras do Sistema de Saúde Suplementar brasileiro.

Para que se comprovasse a robustez do modelo, foram feitas análises de sensibilidade uni e multivariadas. 


\section{Resultado da análise econômica}

Observou-se que o braço de pacientes que incluía o reforço do tratamento local com RIOe apresentou melhor controle local e sobrevida global a um menor custo de tratamento. Foi, portanto, considerado dominante em relação ao braço: radio/quimioterapia + cirurgia de ressecção incompleta (Figura 1), com uma relação de custo-efetividade incremental de -1042.60 reais por ano de vida ganho com qualidade.

As análises de sensibilidade demonstraram que o modelo é robusto e que os resultados apresentados dependem, fundamentalmente, de quanto os resultados de controle local e sobrevida global são melhorados com a RIOe, além do tempo em que os pacientes permanecem em quimioterapia de segunda linha e, tal qual, do custo do tratamento com quimioterapia de segunda linha.

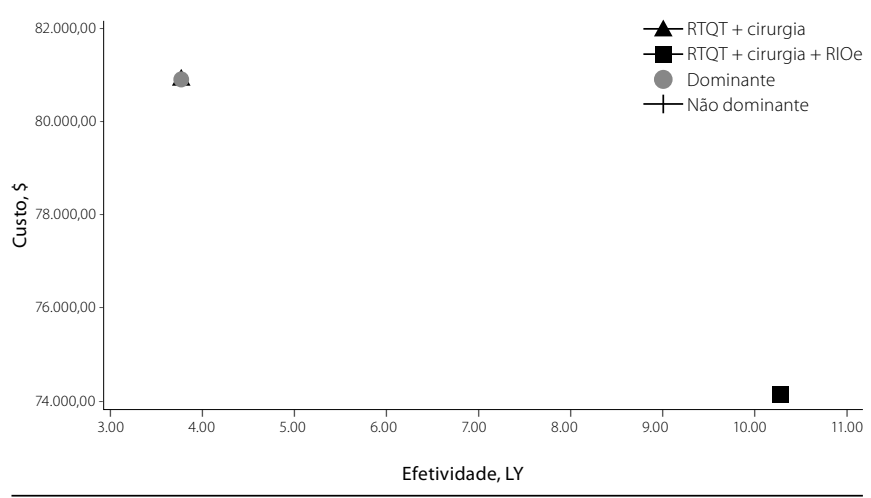

Figura 1. Análise de custo-efetividade

\section{Discussão}

Observamos, a partir dos dados apresentados, que a aplicação da RIOe no tratamento do CRLA, por ocasião de uma cirurgia incompleta (margem comprometida ou doença grosseiramente residual), após a abordagem neoadjuvante padrão, com radioterapia e quimioterapia radiossensibilizante, pode ser uma alternativa interessante para melhorar o controle local dessa enfermidade; e isso, muito provavelmente, vai se reverter em melhora de sobrevida para esse paciente, a um menor custo final total do tratamento. Uma vez que essa estratégia se mostrou dominante, quando comparada à abordagem considerada padrão atualmente (radio/quimioterapia neoadjuvante sem RIOe), ainda que os dados de literatura, nesse cenário clínico, sejam relativamente pobres, e devamos esperar estudos prospetivos e randomizados para discorrermos com mais segurança a respeito dos resultados alcançados. $\mathrm{O}$ autor deste texto entende, no entanto, que, muito provavelmente, tais estudos jamais venham a ser realizados; por dificuldades práticas (o critério de inclusão, neste estudo, depende de resultados cirúrgicos e o procedimento é imediato, o que dificultaria a randomização), pelo relativamente pequeno número de pacientes que, após terapêutica neoadjuvante ótima, venham, infelizmente, a apresentar resposta insatisfatória, impossibilitando a abordagem cirúrgica desejada e, finalmente, pela dificuldade em explicar-se a um doente que, diante de uma doença residual, ele teria, aceitando entrar em tal estudo, uma chance de 50\% de não receber nenhum tratamento local, enquanto o outro grupo receberia uma terapêutica que tem mostrado resultados muito interessantes em estudos não randomizados, como é o caso do estudo utilizado para a confecção desta análise econômica (Sole et al., 2014). Talvez houvesse comprometimento de um princípio básico da ética em ciência, fundamental no desenho de estudos randomizados: a equipoise (Freedman, 1987).

É preciso que se diga que, para a real reprodutibilidade dos resultados apresentados, deve haver seleção adequada do paciente, tanto para o próprio paciente quanto para a operadora de Saúde envolvida no reembolso desse tratamento. $\mathrm{Na}$ ausência de doença residual, estudo anterior, prospectivo e randomizado, realizado na França, mostrou equivalência dos resultados (abordagem neoadjuvante com ou sem RIOe) (Dubois et al., 2011). Nesse caso, segundo a melhor evidência atual, o procedimento não se faz necessário. Não se demonstrou, até o momento, qualquer vantagem comparativa.

Com relação à opção da escolha, para a presente análise, do estudo da radioterapia intraoperatória especificamente realizada com elétrons, em contraposição ao procedimento que pode ser efetuado com fótons, este autor entende que, por serem procedimentos distintos, haja vista os resultados não coincidentes observados no tratamento da neoplasia de mama (Hepel \& Wazer, 2015), eles devem ser objetos de estudos também distintos, ficando as conclusões deste restritas, obviamente, ao procedimento estudado. Para a radioterapia intraoperatória com fótons, nova revisão sistemática deve, então, ser realizada.

Desconhecemos qualquer resultado de estudo semelhante, para a realidade do sistema de saúde privado brasileiro, para que sejam feitas eventuais comparações. Este estudo, por sua vez, apresenta, sem dúvida, algumas debilidades, como, por exemplo, a utilização de dados obtidos a partir de séries de casos não randomizadas e, no caso do grupo controle, revisão sistemática que incluiu estudos retrospectivos. Mas trata-se, muito provavelmente, da melhor evidência disponível, em um cenário que não verá, muito provavelmente, dados os motivos anteriormente elencados, estudos que forneçam nível de evidência mais elevados. Sugere-se, então, que, no cenário nacional, se desenvolvam parcerias entre prestadores e pagadores para que dados de mundo real sejam, futuramente, coletados, e que a precisão deste modelo seja, eventualmente, confirmada, uma vez que os efeitos colaterais da terapêutica são comprovadamente controláveis. O único risco ao qual os pacientes estariam expostos é o de que, futuramente, um be- 
nefício razoavelmente provável não venha, infelizmente, a ser confirmado, o que poderá, a tempo e sem prejuízos relevantes à saúde, ser corrigido prontamente. Dados os robustos resultados apresentados (haja vista as análises de sensibilidade), com a evidência disponível, este autor conclui, então, que estamos diante de uma relação de risco-benefício deveras favorável tanto para o paciente como para a operadora de saúde, e sugere a aplicação desse procedimento, quando factível, no cenário clínico descrito.

\section{Referências bibliográficas}

Agência Nacional de Vigilância Sanitária. CMED: Lista de Preços de Medicamentos - Preços Fábrica e Máximos de Venda ao Governo. Available from: http://portal.anvisa.gov.br/listas-de-precos.

Bray F, Ferlay J, Soerjomataram I, Siegel RL, Torre LA, Jemal A. Global cancer statistics 2018: GLOBOCAN estimates of incidence and mortality worldwide for 36 cancers in 185 countries. CA Cancer J Clin. 2018;68(6):394-424

Calvo FA, Morillo V, Santos M, Serrano J, Gomez-Espí M, Rodriguez M, et al. Interval between neoadjuvant treatment and definitive surgery in locally advanced rectal cancer: impact on response and oncologic outcomes. J Cancer Res Clin Oncol. 2014;140(10):1651-60.

Calvo FA, Meirino RM, Orecchia R. Intraoperative radiation therapy part 2. Clinical results. Crit Rev Oncol Hematol. 2006a;59(2):116-27.

Calvo FA, Serrano FJ, Diaz-González JA, Gomez-Espi M, Lozano E, Garcia R, et al. Improved incidence of pTO downstaged surgical specimens in locally advanced rectal cancer (LARC) treated with induction oxaliplatin plus 5-fluorouracil and preoperative chemoradiation. Ann Oncol. 2006b;17(7):1103-10.

CBHPM - Classificação Brasileira Hierarquizada de Procedimentos Médicos. 2017. Cálculo Básico de Procedimentos Médicos. Available from: http:// www.cbhpm.com.br/wiki/index.php?title=C\%C3\%A1lculo_B\%C3\%A1sico_ de_Procedimentos_M\%C3\%A9dicos.

Dubois JB, Bussieres E, Richaud P, Rouanet P, Becouarn Y, Mathoulin-Pélissier S, et al. Intra-operative radiotherapy of rectal cancer: results of the French multi-institutional randomized study. Radiother Oncol. 2011;98(3):298-303.

Ferenschild FT, Vermaas M, Nuyttens JJ, Graveland WJ, Marinelli AW, van der Sijp JR, et al. Value of intraoperative radiotherapy in locally advanced rectal cancer. Dis Colon Rectum. 2006;49(9):1257-65.

Fletcher GH. Clinical dose-response curves of human malignant epithelial tumours. Br J Radiol. 1973;46(541):1-12.

Freedman B. Equipoise and the ethics of clinical research. N Engl J Med. 1987;317(3):141-5.

Hepel J, Wazer DE. A flawed study should not define a new standard of care. Int J Radiat Oncol Biol Phys. 2015;91(2):255-7.

Inca - Instituto Nacional de Câncer. Estimativa 2020: incidência de câncer no Brasil. 2019. Available from: https://www.inca.gov.br/publicacoes/livros/ estimativa-2020-incidencia-de-cancer-no-brasil.
Kronborg CJS, Jørgensen JB, Petersen JBB, Nyvang Jensen L, Iversen LH, Pedersen BG, et al. Pelvic insufficiency fractures, dose volume parameters and plan optimization after radiotherapy for rectal cancer. Clin Transl Radiat Oncol. 2019;19:72-6.

Kundel Y, Ben Hur R, Shochat Z, Stanislav Z, Fenig E, Brenner B, et al. What is the Best Way to Plan Rectum Three-Dimensional Conformal Radiotherapy in Prone Position-Classic Anatomical Landmark, Three Dimensional Fitting the Planning Target Volume, or Volumetric Modulated Arc? J Med Imaging Radiat Sci. 2020;51(1):103-7.

Kusters M, Dresen RC, Martijn H, Nieuwenhuijzen GA, van de Velde CJ, van den Berg HA, et al. Radicality of resection and survival after multimodality treatment is influenced by subsite of locally recurrent rectal cancer. Int J Radiat Oncol Biol Phys. 2009;75(5):1444-9.

Mirnezami R, Chang GJ, Das P, Chandrakumaran K, Tekkis P, Darzi A, et al. Intraoperative radiotherapy in colorectal cancer: systematic review and meta-analysis of techniques, long-term outcomes, and complications. Surg Oncol. 2013;22(1):22-35.

Owens R, Mukherjee S, Padmanaban S, Hawes E, Jacobs C, Weaver A, et al. Doseescalated intensity-modulated radiotherapy (IMRT) using a simultaneous integrated boost (SIB) in rectal cancer. Ann Oncol. 2019;30 Suppl 4:iv44.

Roeder F, Goetz JM, Habl G, Bischof M, Krempien R, Buechler MW, et al. Intraoperative Electron Radiation Therapy (IOERT) in the management of locally recurrent rectal cancer. BMC Cancer. 2012;12:592.

Sauer R, Becker H, Hohenberger W, Rödel C, Wittekind C, Fietkau R, et al.; German Rectal Cancer Study Group. Preoperative versus postoperative chemoradiotherapy for rectal cancer. N Engl J Med. 2004;351(17):1731-40.

Sauer R1, Liersch T, Merkel S, Fietkau R, Hohenberger W, Hess C, et al. Preoperative versus postoperative chemoradiotherapy for locally advanced rectal cancer: results of the German CAO/ARO/AIO-94 randomized phase III trial after a median follow-up of 11 years. J Clin Oncol. 2012;30(16):1926-33.

Sole CV, Calvo FA, Serrano J, Del Valle E, Rodriguez M, Muñoz-Calero A, et al. Post-chemoradiation intraoperative electron-beam radiation therapy boost in resected locally advanced rectal cancer: long-term results focused on topographic pattern of locoregional relapse. Radiother Oncol. 2014;112(1):52-8.

Valentini V, van Stiphout RG, Lammering G, Gambacorta MA, Barba MC, Bebenek M, et al. Nomograms for predicting local recurrence, distant metastases, and overall survival for patients with locally advanced rectal cancer on the basis of European randomized clinical trials. J Clin Oncol. 2011;29(23):3163-72.

Wells BJ, Stotland P, Ko MA, Al-Sukhni W, Wunder J, Ferguson P, et al. Results of an aggressive approach to resection of locally recurrent rectal cancer. Ann Surg Oncol. 2007;14(2):390-5.

Willett CG, Shellito PC, Tepper JE, Eliseo R, Convery K, Wood WC. Intraoperative electron beam radiation therapy for primary locally advanced rectal and rectosigmoid carcinoma. J Clin Oncol. 1991;9(5):843-9.

Yu TK, Bhosale PR, Crane CH, lyer RB, Skibber JM, Rodriguez-Bigas MA, et al. Patterns of locoregional recurrence after surgery and radiotherapy or chemoradiation for rectal cancer. Int J Radiat Oncol Biol Phys. 2008;71(4):1175-80. 